\title{
Kahweol inhibits lipid accumulation and induces Glucose-uptake through activation of AMP-activated protein kinase (AMPK)
}

\author{
Jung-Hwan Baek ${ }^{1,2}$, Nam-Jun Kim ${ }^{1,2}$, Jun-Kyu Song ${ }^{1,2}$ \& Kyung-Hee Chun ${ }^{1,2, *}$ \\ ${ }^{1}$ Department of Biochemistry and Molecular Biology, Yonsei University College of Medicine, ${ }^{2}$ Brain Korea 21 PLUS Project for Medical \\ Science, Yonsei University, Seoul 03722, Korea
}

\begin{abstract}
Weight loss $\geq 5$ percent is sufficient to significantly reduce health risks for obese people; therefore, development of novel weight loss compounds with reduced toxicity is urgently required. After screening of natural compounds with antiadipogenesis properties in 3T3-L1 cells, we determined that kahweol, a coffee-specific diterpene, inhibited adipogenesis. Kahweol reduced lipid accumulation and expression levels of adipogenesis and lipid accumulation-related factors. Levels of phosphorylated AKT and phosphorylated JAK2, that induce lipid accumulation, decreased in kahweol-treated cells. Particularly, kahweol treatment significantly increased AMPactivated protein kinase (AMPK) activation. We revealed that depletion of AMPK alleviated reduction in lipid accumulation from kahweol treatment, suggesting that inhibition of lipid accumulation by kahweol is dependent on AMPK activation. We detected more rapid reduction in blood glucose levels in mice administrated kahweol than in control mice. We suggest that kahweol has anti-obesity effects and should be studied further for possible therapeutic applications. [BMB Reports 2017; 50(11): 566-571]
\end{abstract}

\section{INTRODUCTION}

Obesity is a disease that is becoming a global issue. Obesity increases the risk of mortality and morbidity, because it is accompanied by hypertension, heart disease, type 2 diabetes, and some types of cancer (1). Pharmacotherapeutic drugs have been developed and used for treating obesity. According to meta-analysis about effects of anti-obesity drugs approved by

*Corresponding author. Tel: +82-2-2228-1699; Fax: +82-2-3125041; E-mail: khchun@yuhs.ac

https://doi.org/10.5483/BMBRep.2017.50.11.031

Received 11 March 2017, Revised 12 April 2017, Accepted 8 June 2017

Keywords: Adipogenesis, AMPK, Coffee bean, Glucose-uptake, Kahweol the Food and Drug Administration (FDA), anti-obesity drugs promote moderate weight loss (2). Initially, the thyroid hormone was selected as a therapeutic target, and Amphetamine became popular for an appetite suppressant in the late 1930s (3). Sibutramine was approved for long-term treatment in 1997. Orlistat was approved by the FDA for long-term treatment in 1999. It reduces intestinal fat absorption by inhibiting gastric and pancreatic lipases that hydrolyze triglyceride. The latest approved anti-obesity drugs were liraglutide and bupropion-naltrexone in 2014 (3).

The Endocrine Society suggests use of approved anti-obesity drugs to preserve long-term weight improves complications of obesity and increases adaptation to behavioral modification (4). However, safety concerns for anti-obesity drugs have emerged, and some drugs have been removed from the market. For example, dexfenfluramine, associated with cardiovascular side effects was withdrawn from FDA approval in 1997. Sibutramine also provokes severe cardiovascular events (5). Moreover, even with current drugs on the market such as orlistat, bupropion-naltrexone, phentermine-topiramate and liraglutide, treatment decisions are driven by co-existing medical conditions (6). For example, liraglutide may be a more relevant agent in individuals with type 2 diabetes because it lowers blood glucose levels. The use of bupropionnaltrexone in patients with alcohol or opioid dependence is associated with neuropsychiatric complications (7). Finally, the U.S. Drug Enforcement Administration (DEA) has classified most anti-obesity drugs as controlled substances, and many states have passed strict regulations relative to prescriptions and use.

Even if only 5 percent of weight is lost, the risk of metabolic disorders such as type 2 diabetes, non-alcoholic fatty liver disease or hypertension is significantly reduced (8). The goal of anti-obesity drugs is to enable weight loss of more patients. A barrier to drug approval is not only individuals with health risks but also healthy individuals that may use anti-obesity drugs which may lead to serious side effects. Low side-effects from drugs are essential for approval $(1,9,10)$.

Consequently, the primary objective of our research was to identify anti-obesity drugs by screening natural products, 
without significant side effects. We used a library using in clinic with other purpose to cure diseases, such as antibiotics and anti-cancer agents. We established a screening method to determine lipid accumulation using 3T3-L1 cells. After screening with natural products, we identified kahweol, that is a diterpene found in beans of Coffea arabica and structurally related to cafestol (Fig. 1A and B). Recent research suggests that kahweol may have beneficial effects on bones by inhibiting osteoclast differentiation (11). Another recent study revealed that kahweol has anti-inflammatory and antiangiogenic effects (12), offering a possible mechanism for epidemiological studies revealing a relationship between unfiltered coffee intake and decreased risk of cancer.

In this study, we examined kahweol's effect on adipocyte differentiation and lipid accumulation. Because AMP-Activated Protein Kinase (AMPK) is an enzyme regulating glucose transport and lipid metabolism, AMPK is a therapeutic target of diabetic agent anti-and obesity (13). Interestingly, we determined that kahweol has a potent effect on activation of AMPK, thereby increasing glucose uptake in blood of sugar-taken
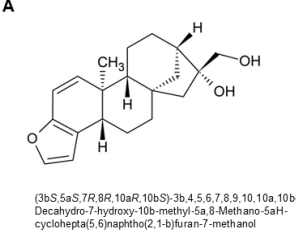

c

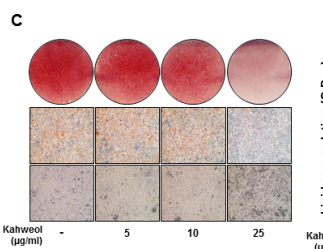

D

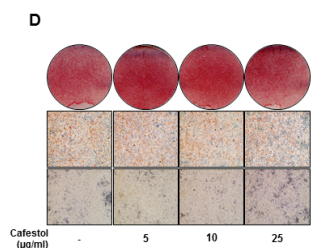

Catesta

Fig. 1. Kahweol inhibits lipid accumulation in 3T3-L1 cells, whereas cafestol has no effect on lipid accumulation. (A) Structure of kahweol. (B) Structure of cafestol. (C, D) Oil Red O staining of kahweol- and cafestol-treated 3T3-L1 cells. After MDI induction, 3T3-L1 cells were treated with kahweol or cafestol on days 2-6. Oil Red O staining was performed on day 6. Measurement of lipid accumulation. Stained ORO was eluted with 100 percent isopropanol and measured using the OD500. ${ }^{* * * P}<0.001$, DMSO vs. kahweol, DMSO vs. cafestol. (E) Cell viability assays. Confluent 3T3-L1 cells were treated with kahweol for 48 hours. (F) Protein expression of PPAR $\gamma, \mathrm{C} / \mathrm{EBP} \alpha, \mathrm{FABP} 4$, and FASN was detected by western blotting. Protein expression was normalized to $\beta$-actin. mice. We suggest that kahweol may be an ideal agent for obesity attenuation and suggest clinical trials further examine potential applications.

\section{RESULTS}

\section{Kahweol reduces lipid accumulation in 3T3-L1 cells}

Excessive increase of adipocyte number (hyperplasia) and adipocyte size (hypertrophy) contributes to obesity. In adults, obesity is caused by an increase in adipocyte size in white adipose tissue from excessive storage of triglyceride. Inhibition of triglyceride accumulation in adipocytes is a promising strategy for prevention and treatment of obesity. To not influence mitotic clonal expansion, a period when cells proliferate during adipocyte differentiation, 3T3-L1 cells were treated with different concentration of kahweol 2 days after MDI-induction. Lipid accumulation in 3T3-L1 cells was measured using Oil Red O staining on day 6 (Fig. 1C). Kahweol $(25 \mu \mathrm{g} / \mathrm{ml})$ significantly reduced lipid accumulation, whereas cafestol did not reduce lipid accumulation (Figs. 1C and D). To confirm the inhibitory effect of kahweol on lipid accumulation is not a result of cell cytotoxicity, a cell viability assay was conducted. We confirmed there was no difference in cell viability (Fig. 1E). We tested if kahweol treatment affects protein expression of PPAR $\gamma, \mathrm{C} / \mathrm{EBP} \alpha, \mathrm{FABP} 4$, and FASN, that regulate adipocyte differentiation and lipid metabolism.
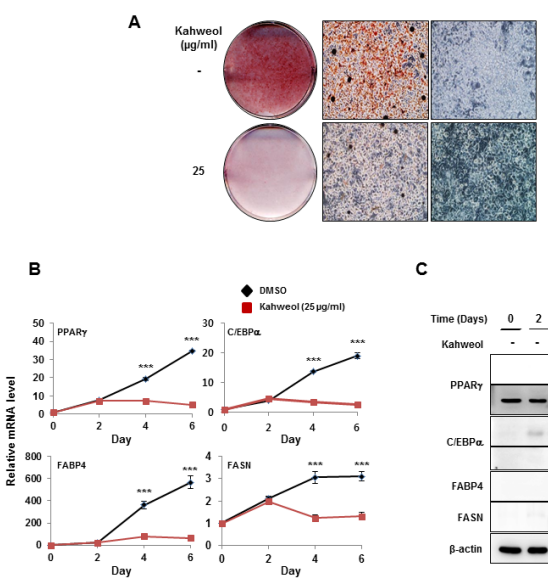

Fig. 2. Kahweol attenuates expression of adipogenic factors. (A) Oil Red O staining of kahweol-treated 3T3-L1 cells. After MDI induction, 3T3-L1 cells were treated with kahweol on days 2-6. Oil Red $\mathrm{O}$ staining was conducted on day 6. (B) mRNA expression of PPAR $\gamma, \mathrm{C} / \mathrm{EBP} \alpha, \mathrm{FABP} 4$, and FASN was detected by real-time PCR. RNA samples were prepared on days $0,2,4$, and 6. 3T3-L1 cells were treated with kahweol on days 2-6. mRNA expression was normalized to $\beta$-actin. $* * * P<0.001$, DMSO vs. kahweol. (C) Protein expression of PPAR $\gamma, \mathrm{C} / \mathrm{EBP} \alpha, \mathrm{FABP} 4$, and FASN was detected by western blotting. Protein samples were prepared on days $0,2,4$, and 6. 3T3-L1 cells were treated with kahweol on days 2-6. Protein expression was normalized to $\beta$-actin. 
Kahweol significantly reduced expression of these proteins in a dose-dependent manner (Fig. 1F).

\section{Kahweol suppresses the expression of adipogenesis and lipid accumulation-related genes in 3T3-L1 cells}

We identified that $25 \mu \mathrm{g} / \mathrm{ml}$ kahweol has an inhibitory effect on lipid accumulation through dose-dependent treatment (Fig. 2A). To examine how kahweol treatment affects adipogenesis and lipid accumulation-related genes during adipocyte differentiation, cell lysates were prepared on days $0,2,4$, and 6. We treated cells with kahweol 2 days after adipogenic stimuli, focusing on the effect of kahweol on lipid accumulation. Kahweol significantly repressed mRNA (Fig. 2B) and protein expression (Fig. 2C) of PPAR $\gamma, \mathrm{C} / \mathrm{EBP} \alpha, \mathrm{FABP} 4$, and FASN after 2 days of treatment, suggesting that kahweol affects adipogenesis and lipid accumulation.

\section{Kahweol reduces MDI-induced adipocyte differentiation and lipid droplet size}

To demonstrate the effect of kahweol on MDI-induced adipogenesis, 3T3-L1 cells were treated with MDI and kahweol at day 0 . Kahweol treatment reduced adipocyte differentiation and lipid accumulation (Fig. 3A). We also examined the effect of kahweol on late adipocyte differentiation. 3T3-L1 cells were incubated with kahweol from days 6 to 10. Kahweol treatment slightly reduced lipid accumulation, and we revealed lipid droplet size in kahweol-treated 3T3-L1 cells was smaller than that of control cells (Fig. 3B).

To identify kahweol's effect on MDI-induced mitotic clonal
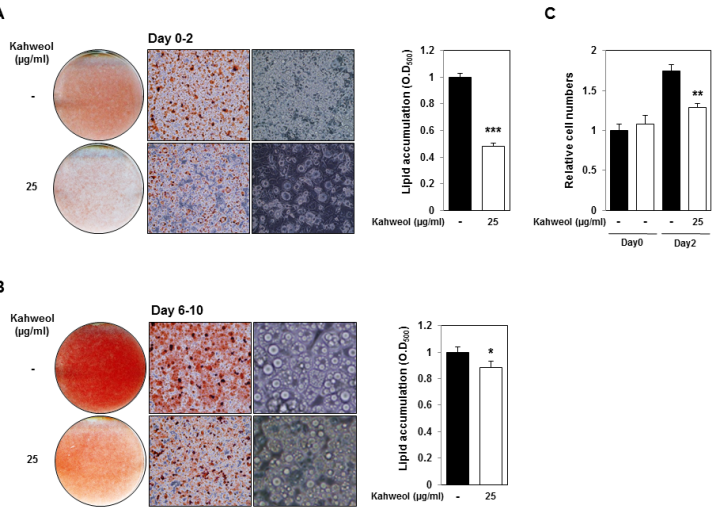

Fig. 3. Kahweol inhibits mitotic clonal expansion and reduces lipid droplet size. (A) Oil Red O staining and lipid accumulation of kahweol-treated 3T3-L1 cells. 3T3-L1 cells were treated with MDI and kahweol for day 2. Oil Red O staining was conducted on day 6. (B) Oil Red $\mathrm{O}$ staining and lipid accumulation of kahweol-treated 3T3-L1 cells. 3T3-L1 cells were treated with kahweol on days 6-10. Oil Red O staining was conducted on day 10. (C) Increase of cell numbers during mitotic clonal expansion. 3T3-L1 cells were treated with DMI and kahweol until day 2 , and cell numbers were measured on day 0 and 2 . ${ }^{*} \mathrm{P}<$ $0.05, * * P<0.01, * * * P<0.001$, untreated control vs. kahweol. expansion, we measured cell number on day 0 and 2. On day 2, vehicle-treated 3T3-L1 cell number increased about 1.8-fold compared with that in day 0. Kahweol-treated 3T3-L1 cell number also increased, compared with that at day 0 , but increased significantly less than vehicle-treated samples (Fig. 3C). Data indicate that kahweol represses adipocyte differentiation through inhibition of mitotic clonal expansion and reduces lipid droplet size.

\section{Kahweol inhibits lipid accumulation and increase glucose} uptake through activation of AMPK

Many studies have reported that kahweol suppresses activation

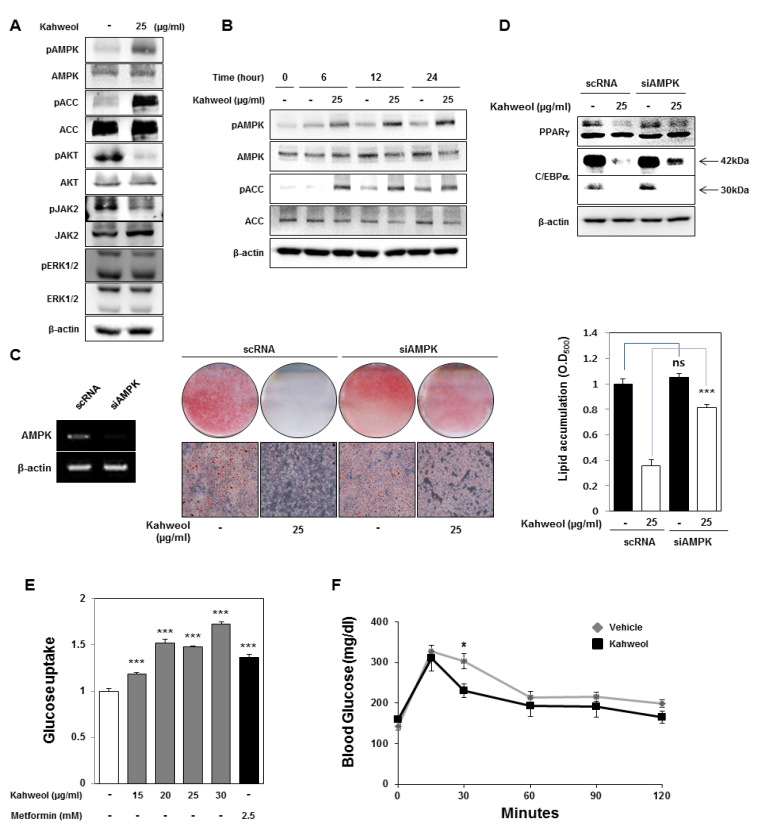

Fig. 4. Kahweol reduces lipid accumulation and increase glucose uptake through activation of AMPK. (A) Western blot analysis of signal transduction-related proteins. 3T3-L1 cells were treated with kahweol on day 2 and incubated for 24 hours. (B) Activity of signal transduction-related proteins, such as AMPK and ACC was detected by western blotting. 3T3-L1 cells were treated with kahweol on day 2. (C) Knockdown of AMPKa1 using small interfering RNA (siRNA). After transfection of AMPKa1 siRNA, 3T3-L1 cells were incubated with MDI. Then, 3T3-L1 cells were treated with kahweol on day 2. $* * * P<0.001$, scRNA vs. siAMPK. (D) Protein expression of PPAR $\gamma$ and $\mathrm{C} / \mathrm{EBP} \alpha$ was detected by western blotting. Protein samples were prepared on day 4. (E) Glucose uptake analysis. 3T3-L1 cells were incubated with kahweol or metformin for 48 hours. The remaining level of glucose in media was measured using a glucose assay kit. Metformin was used as a positive control. $* \mathrm{P}<0.05, * * \mathrm{P}<0.01, * * * \mathrm{P}<0.001$, untreated control vs. kahweol, untreated control vs. metformin (F) Glucose tolerance test ( $\mathrm{n}=5$ for each group). 8 week-old $\mathrm{C} 57 \mathrm{BL} / 6$ mice were treated with the vehicle (DMSO) or kahweol $(100 \mathrm{mg} / \mathrm{kg}$ ) every 2 days via oral administration. After 2 weeks, the glucose tolerance test was conducted at $0,15,30,60,90$, and 120 minutes. ${ }^{*} \mathrm{P}<0.05$, vehicle $(\mathrm{n}=5)$ vs. kahweol $(\mathrm{n}=5)$. 
of AKT and ERK1/2 $(11,14)$. AKT and ERK1/2 pathways were reported to regulate adipocyte maturation (15). We examined changes in activation of these signaling pathways that are involved in adipocyte differentiation and lipid metabolism (Fig. 4A). Phosphorylation of AKT and JAK2 decreased after kahweol treatment; in contrast, phosphorylation of ERK1/2 was not affected by kahweol treatment. Interestingly, we found that kahweol treatment significantly increased the phosphorylation of AMPK and its downstream target Acetyl-CoA carboxylase (ACC). We also validated that kahweol treatment increased phosphorylated AMPK and ACC in a time-dependent manner (Fig. 4B). Many studies have reported that AMPK is activated by phosphorylation and that it inhibits adipocyte differentiation and increases glucose uptake and fatty acid oxidation (13).

We investigated if kahweol may inhibit lipid accumulation through AMPK activation. The inhibitory effect of kahweol on lipid accumulation was alleviated by knockdown of AMPK using siRNAs (Fig. 4C). Knockdown of AMPK also reduced the effect of kahweol on expression of adipogenesis and lipid metabolism-related genes (Fig. 4D). These results suggest that kahweol suppresses lipid accumulation by up-regulation of AMPK activation. Activation of AMPK promotes cellular glucose uptake through glucose transporter. Metformin known as AMPK activator has an anti-diabetic effect (16). Since kahweol also has the effect of AMPK activation as metformin, we hypothesized that kahweol may reveal an anti-diabetic effect. In 3T3-L1 cells, kahweol treatment elevated glucose uptake in a dose-dependent manner (Fig. 4E). In addition, we tested if kahweol may improve glucose homeostasis in vivo. 8 week-old mice were administered kahweol every 2 days by oral gavage. After 2 weeks, we conducted glucose tolerance test (GTT). Clearance of blood glucose was faster in kahweol-treated mice than in control mice (Fig. 4F).

\section{DISCUSSION}

Coffee, one of the most consumed beverages globally contains a variety of ingredients that are beneficial to health. Epidemiological studies suggest that coffee consumption may reduce incidence of several chronic diseases such as obesity, type 2 diabetes, neurodegenerative diseases, Parkinson's disease, and cardiovascular diseases (17-19). Many studies have revealed that coffee consumption decreases accumulation of lipid and collagen in the liver and modulates antioxidant and inflammatory responses. However, mechanisms for its beneficial effects are not fully understood. Coffee intake is known to increase cholesterol and the diterpenes cafestol and kahweol are implicated in its effect (20). Cafestol and kahweol cause extracellular accumulation of LDL by reducing activity of hepatic LDL receptors (21).

In this study, we determined the molecular mechanism by which kahweol inhibits lipid accumulation in 3T3-L1 cells. First, we established a screening method using adipogenesis of
3T3-L1 cells. We treated cells with selected compounds from natural compound libraries 2 days after initiation of adipocyte differentiation, then quantified lipid accumulation in adipogenic 3T3-L1 cells by Oil Red staining. Among these compounds, we found that kahweol, a coffee-specific diterpene, has a strong anti-lipogenic effect compared to its structural analog cafestol. Kahweol inhibited lipid accumulation and adipocyte differentiation, but cafestol had no effect on these in 3T3-L1 cells. Many studies on kahweol have defined its inhibitory effects on cancer, angiogenesis, and inflammation. For example, kahweol inhibits cell growth by inducing proteasomal degradation of cyclin D1 via ERK1/2, JNK, and GKS3 $\beta$ dependent phosphorylation (22) and HSP 70 Expression (23) in human colorectal cancer. Kahweol induces apoptosis via inhibition of STAT3 phosphorylation in human lung adenocarcinoma A549 cells (24). The anti-angiogenic effect of kahweol on endothelial cells was demonstrated by inhibition of MMP-2 and UPA expression (12) and inhibition of VEGFR2 signaling pathway (25). We detected that kahweol treatment reduced the expression of adipogenic factors, such as PPAR $\gamma$ and $\mathrm{C} / \mathrm{EBP} \alpha$, that induce adipogenic differentiation, lipid synthesis related factor, FASN, and the lipid accumulation promoting factor FABP4.

Interestingly, we determined that kahweol induces activation of AMPK. AMPK is a serine/threonine kinase, that is a key enzyme for maintaining cellular energy homeostasis $(13,26)$. AMPK has a heterotrimer complex consisting $\alpha, \beta$ and $\gamma$ subunits. Catalytic $\alpha$ subunit contains Thr172 phosphorylated by AMPK upstream kinase. Regulatory $\gamma$ subunit has four Cystathionine $\beta$ synthase (CBS) domains that create two AMP binding sites known as the Bateman domain. One of major roles of AMPK is the regulation of lipid metabolism. AMPK activation phosphorylates and inactivates acetyl-CoA carboxylase, an enzyme involved in fatty acid synthesis, and consequently inhibits synthesis of fatty acid and increases $\beta$-oxidation. AMPK also regulates glucose metabolism. AMPK increases glycolysis by activating of 6-phosphofructo-2-kinase/ fructose-2, 6-bisphosphatase $2 / 3$ and suppresses glycogen synthesis through inhibition of glycogen synthase (13). AMPK increases glucose uptake by promoting glucose transporter 4 and hexokinase 2 expressions in skeletal muscle cells $(27,28)$. AMPK modulates various metabolic processes and its dysregulation is commonly observed in type 2 diabetes, obesity, and several types of cancer. AMPK activation is a promising therapeutic strategy for alleviating metabolic dysfunction. Many studies have revealed that AMPK activators inhibit adipocyte differentiation in vitro and ameliorate adiposity in high-fat diet-fed obese mice (29-31). Metformin, a typical AMPK activator is an anti-diabetic drug for therapy of type 2 diabetes (16).

In this study, kahweol induces phosphorylation of AMPK. The effect of kahweol treatment on reducing lipid accumulation was decreased by depletion of AMPK, suggesting that the inhibitory effect of kahweol on lipid accumulation was due to 
phosphorylation of AMPK. Mice were fasted for 15 hours and then fed glucose. The blood glucose level of kahweol-pretreated mice decreased faster than untreated mice. However, we do not know how kahweol increases phosphorylation of AMPK. Thr172 phosphorylation of the AMPK $\alpha$-subunit is regulated by three phosphatases: $\mathrm{Mg}^{2+} / \mathrm{Mn}^{2+}$ dependent protein phosphatase 1E (PPM1E), protein phosphatase 2A (PP2A), and protein phosphatase $2 \mathrm{C}$ (PP2C) $(13,32)$. If AMP/ATP and ADP/ATP ratios are low, phosphatases access and dephosphorylate Thr172 of AMPK $\alpha$-subunit. Thr172 phosphorylation of the AMPK $\alpha$-subunit is also regulated by three upstream AMPK kinases: liver kinase B1 (LKB1), calcium/calmodulindependent kinase 2 (CaMKK2) (33), and TGF $\beta$-activated kinase 1 (TAK1) (34). When intracellular energy is low, level of AMP and ADP elevates and, AMP and ADP bind to Bateman domains of the AMPK $\gamma$-subunit. This leads to conformational change that exposes the catalytic domain of AMPK $\alpha$-subunit, and prevents access to phosphatases. Residue Thr172 in catalytic domain of AMPK $\alpha$-subunit is phosphorylated by upstream AMPK kinases. AMPK activation is regulated allosterically by competitive binding of ATP and AMP or ADP. Currently, we are studying how kahweol regulates phosphorylation of AMPK by examining AMPK-related signaling pathways and/or the kinases that are regulated by kahweol.

Together, we suggest that kahweol has anti-obesity effects and should be studied further for possible therapeutic applications.

\section{MATERIALS AND METHODS}

Materials and methods are available in supplementary information.

\section{ACKNOWLEDGEMENTS}

This work was supported by the National Research Foundation of Korea (NRF) grant funded by the Korea government (MSIP) (NRF-2014R1A2A1A11050600), the Bio \& Medical Technology Development Program of the NRF funded by the Korean government, MSIP (NRF-2015M3A9B6073835, NRF-2015M3 A9B6073833), the Basic Science Research Program through the National Research Foundation of Korea (NRF) funded by the Ministry of Education (NRF-2014R1A1A2055009) and by the National Research Foundation of Korea (NRF) grants funded by the Korean government (Ministry of Science, ICT and Future Planning) (NRF-2016R1A6A3A11935119).

\section{CONFLICTS OF INTEREST}

The authors have no conflicting interests.

\section{REFERENCES}

1. Bray GA (2013) Why do we need drugs to treat the patient with obesity? Obesity (Silver Spring) 21, 893-899

2. Li Z, Maglione M, Tu W et al (2005) Meta-analysis: pharmacologic treatment of obesity. Ann Intern Med 142, 532-546

3. Xia Y, Kelton CM, Guo JJ, Bian B and Heaton PC (2015) Treatment of obesity: Pharmacotherapy trends in the United States from 1999 to 2010. Obesity (Silver Spring) 23, 1721-1728

4. Apovian CM, Aronne LJ, Bessesen DH et al (2015) Pharmacological management of obesity: an endocrine Society clinical practice guideline. J Clin Endocrinol Metab 100, 342-362

5. Cheung BM, Cheung TT and Samaranayake NR (2013) Safety of antiobesity drugs. Ther Adv Drug Saf 4, 171-181

6. Khera R, Murad MH, Chandar AK et al (2016) Association of Pharmacological Treatments for Obesity With Weight Loss and Adverse Events: A Systematic Review and Meta-analysis. JAMA 315, 2424-2434

7. Yanovski SZ and Yanovski JA (2014) Long-term drug treatment for obesity: a systematic and clinical review. JAMA 311, 74-86

8. Goldstein DJ (1992) Beneficial health effects of modest weight loss. Int J Obes Relat Metab Disord 16, 397-415

9. Choi HY, Saha SK, Kim K et al (2015) G protein-coupled receptors in stem cell maintenance and somatic reprogramming to pluripotent or cancer stem cells. BMB Rep 48, 68-80

10. Kang $\mathrm{H}$ and Hata $\mathrm{A}$ (2015) The role of microRNAs in cell fate determination of mesenchymal stem cells: balancing adipogenesis and osteogenesis. BMB Rep 48, 319-323

11. Fumimoto R, Sakai E, Yamaguchi $Y$ et al (2012) The coffee diterpene kahweol prevents osteoclastogenesis via impairment of NFATc1 expression and blocking of Erk phosphorylation. J Pharmacol Sci 118, 479-486

12. Cárdenas C, Quesada AR and Medina MA (2011) Medina, Anti-angiogenic and anti-inflammatory properties of kahweol, a coffee diterpene. PLoS One 6, e23407

13. Jeon SM (2016) Regulation and function of AMPK in physiology and diseases. Exp Mol Med 48, e245

14. Oh JH, Lee JT, Yang ES et al (2009) The coffee diterpene kahweol induces apoptosis in human leukemia U937 cells through down-regulation of Akt phosphorylation and activation of JNK. Apoptosis 14, 1378-1386

15. Rosen ED and MacDougald OA (2006) MacDougald Adipocyte differentiation from the inside out. Nat Rev Mol Cell Biol 7, 885-896

16. Zhang BB, Zhou G and Li C (2009) AMPK: an emerging drug target for diabetes and the metabolic syndrome. Cell Metab 9, 407-416

17. Morisco F, Lembo V, Mazzone G, Camera S and Caporaso N (2014) Coffee and liver health. J Clin Gastroenterol 48 Suppl 1, S87-90

18. Higdon JV and Frei B (2006) Coffee and health: a review of recent human research. Crit Rev Food Sci Nutr 46, 101-123

19. Kim AR, Yoon BK, Park $\mathrm{H}$ et al (2016) Caffeine inhibits adipogenesis through modulation of mitotic clonal expansion and the AKT/GSK3 pathway in 3T3-L1 adipocytes. BMB Rep 49, 111-115

20. Gross G, Jaccaud E and Huggett AC (1997) Analysis of the 
content of the diterpenes cafestol and kahweol in coffee brews. Food Chem Toxicol 35, 547-554

21. Rustan AC, Halvorsen B, Huggett AC, Ranheim $T$ and Drevon CA (1997) Effect of coffee lipids (cafestol and kahweol) on regulation of cholesterol metabolism in HepG2 cells. Arterioscler Thromb Vasc Biol 17, 21402149

22. Park GH, Song HM and Jeong JB (2016) The coffee diterpene kahweol suppresses the cell proliferation by inducing cyclin D1 proteasomal degradation via ERK1/2, JNK and GKS3beta-dependent threonine-286 phosphorylation in human colorectal cancer cells. Food Chem Toxicol 95, 142-148

23. Choi DW, Lim MS, Lee JW et al (2015) The Cytotoxicity of Kahweol in HT-29 Human Colorectal Cancer Cells Is Mediated by Apoptosis and Suppression of Heat Shock Protein 70 Expression. Biomol Ther (Seoul) 23, 128-133

24. Kim HG, Hwang YP and Jeong HG (2009) Kahweol blocks STAT3 phosphorylation and induces apoptosis in human lung adenocarcinoma A549 cells. Toxicol Lett $187,28-34$

25. Moeenfard M, Cortez A, Machado V et al (2016) Anti-Angiogenic Properties of Cafestol and Kahweol Palmitate Diterpene Esters. J Cell Biochem 117, 27482756

26. Liu XL, Ming YN, Zhang JY, Chen XY, Zeng MD and Mao YM (2017) Gene-metabolite network analysis in different nonalcoholic fatty liver disease phenotypes. Exp Mol Med 49, e283

27. McGee SL, van Denderen BJ, Howlett KF et al (2008)
AMP-activated protein kinase regulates GLUT4 transcription by phosphorylating histone deacetylase 5 . Diabetes $57,860-867$

28. Stoppani J, Hildebrandt AL, Sakamoto K, Cameron-Smith D, Goodyear LJ and Neufer PD (2002) AMP-activated protein kinase activates transcription of the UCP3 and HKII genes in rat skeletal muscle. Am J Physiol Endocrinol Metab 283, E1239-E1248

29. Habinowski SA and Witters LA (2001) The effects of AICAR on adipocyte differentiation of 3T3-L1 cells. Biochem Biophys Res Commun 286, 852-856

30. Figarola JL and Rahbar S (2013) Smallmolecule COH-SR4 inhibits adipocyte differentiation via AMPK activation. Int J Mol Med 31, 1166-1176

31. Kim J, Yang G, Kim Y, Kim J and Ha J (2016) AMPK activators: mechanisms of action and physiological activities. Exp Mol Med 48, e224

32. Davies SP, Helps NR, Cohen PT and Hardie DG (1995) 5'-AMP inhibits dephosphorylation, as well as promoting phosphorylation, of the AMP-activated protein kinase. Studies using bacterially expressed human protein phosphatase-2C alpha and native bovine protein phosphatase2AC. FEBS Lett 377, 421-425

33. Hardie DG (2008) AMPK: a key regulator of energy balance in the single cell and the whole organism. Int J Obes (Lond) 32, Suppl 4: S7-12

34. Herrero-Martín G, Høyer-Hansen M, García-García C et al (2009) TAK1 activates AMPK-dependent cytoprotective autophagy in TRAIL-treated epithelial cells. EMBO J 28, 677-685 